\title{
Real-Time Implementation of Passive Fault- Tolerant Speed Control for Unloading MS150 DC Motor System
}

\author{
Katherin Indriawati* and Rahajeng Kurnianingtyas \\ Department of Engineering Physics, Institut Teknologi Sepuluh Nopember, \\ Jl. Teknik Kimia, Sukolio, Surabaya 60111, Indonesia
}

\begin{abstract}
The failure of a DC motor in an electric car caused by an error in the actuator and sensor can endanger the driver and the surrounding environment. This research focuses on the design of a Passive Fault Tolerant Control (PFTC) system that can work when an actuator and sensor error occurs in the MS150 DC modular servo system speed control. The first step is to identify the MS150 DC modular servo system. The second step is to design a state feedback control system with an integrator. The third step is to design a simulation PFTC system and design a PFTC system to be implemented in realtime. To find out the performance of the designed PFTC system, a test is carried out with a bias error of about $0.1 \mathrm{~V}$ to $0.8 \mathrm{~V}$. On the results of implementing the PFTC system in real-time on the simulation error, the maximum overshoot value is smaller than the system without PFTC, besides the application of AFTC causes the resulting settling time is smaller than the system without PFTC.
\end{abstract}

Keywords: Actuators, sensors, PFTC Systems, MS150 DC modular servo systems.

\section{Introduction}

Currently, the use of electric cars has increased along with public awareness of the environment. The main component of an electric car is a DC motor. In electric vehicles, DC motors work like a servo system with motor speed according to temporary requirements of changes in load torque. In this control system, the sensors and actuators are very sensitive and easily damaged [1]. The minor fault that occurs on both should be removed as early as possible to prevent system failure. On the other hand, PID controllers are usually used in system controls but they have poor performance when the load changes or motor dynamics [2]. Therefore, the system requires control that can tolerate fault on certain limits.

Fault-Tolerant Control (FTC) is a control system that can tolerate fault that occurs and maintain the system performance can be maintained. The purpose of the FTC is to ensure that the system can operate correctly even if a fault occurs. The FTC can be classified into two types, namely Passive Fault Tolerance Control (PFTC) and Active Fault Tolerance Control (AFTC) [3]. AFTC responds fault that occurs on the system by redesigning controller action based on the scheme of Fault Detection and Isolation (FDD) scheme. While PFTC is designed to make the control system are robust against fault without requiring the identification process fault that happens [1, 4]. PFTC has the advantage of

*Corresponding author: kindriawati@gmail.com 
being able to work well on systems that have high uncertainty. FTC can be used with different objectives according to controlled applications, for example, aircraft safety and costs in industrial processes [5]. The robust control system is a control system that can work well even though there are disturbances in the system with unknown characteristics. The robust theory works by calculating system uncertainty. Controller parameters on robust controls are fixed and designed using the assumption of a certain maximum level of disturbance.

The robust control system had been applied to the servo system [6]. The controller works with the assumption that there is no fault in the measurement so that the proposed control system cannot work properly when a sensor fault occurs. Fault actuator can be seen as a disturbance on robust controls. However, the amount of disturbance that can be accommodated is only limited to the value of the assumptions set at the time of design, so it cannot resolve with larger actuator faults. Besides, the condition of motors operates with load change causes a change in the system dynamics, making it more difficult to control if only using linear time-invariant (LTI) model approach in designing robust controls.

Several previous research had already been done. In the 2002, research on robust control without observers was done. This research discusses the robust control applied to DC motors with fault focusing on a disturbance at the moment of inertia [6]. Using robust controller state feedback with analysis of the LMI approach is stated to be very flexible and efficient because it can maintain system performance. However, the research only observed the moment of inertia disturbances and has not reviewed the fault that occurs in the sensor and/or actuator components.

The development of the next research done in 2005 by using observers on fault detection isolation (FDI) to compensate for sensor and actuator faults [7]. The method chosen is AFTC and applied to DC motor speed control. The results showed that faults were detected and compensated. However, the researchers concluded that the research had not considered FDI delay which may happen often, so it affects the stability and performance of the system.

The use of observer to compensate faults without further FDI mechanisms was done [8]. Application of the FTC in the case of a DC motor is done by considering sensor and actuator faults and disturbance. In this case, the observer is designed to be robust against disturbance, using the LMI approach. Although the controller in charge of tracking the setpoint, however, in this research have not implemented a thorough analysis of the integration of controller and observer. So, if the controller is robust, then the possible faults can occur that are not detected by the observer. This can affect the results of the required compensation and the DC motor speed response.

Based on previous research studies that have been described previously, this research is proposed. This research will design a PFTC on a DC servo motor system for speed control with actuator and sensor faults. The PFTC method was chosen because this method is not through the FDI mechanism so that it can minimize the delay in the fault estimation process. Robust systems will be designed include all parts of the control system so that the system can run optimization more optimal.

\section{System description}

\subsection{Modular DC motor servo system}

The DC motor is an electrical machine that has changed the working principle of convert electrical energy into mechanical energy. Energy conversion is the implementation of Lorentz law, which is the law that applies to electricity in the conductive wire in the magnetic field [9]. MS150DC modular servo system is a set of electronic blocks that are used to control the speed and position of the DC motor. 


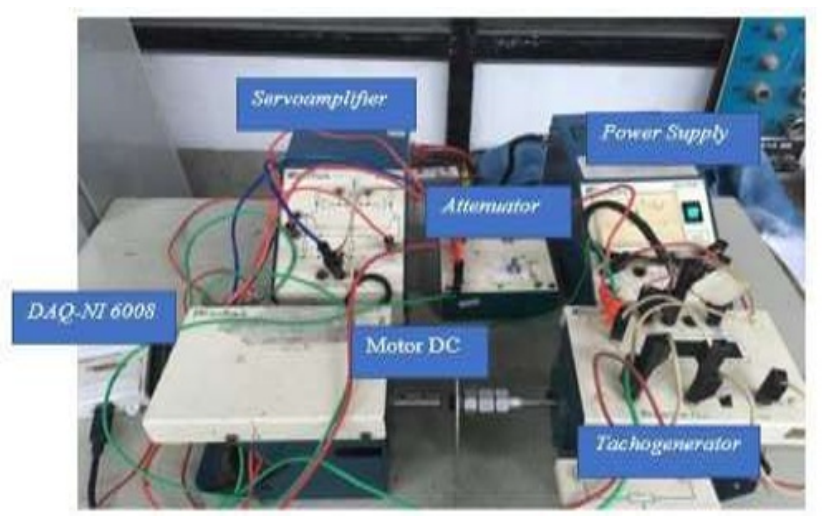

Fig 1. The modular servo system MS150 DC

Figure 1 represents the image of the modular servo system that consists of a power supply, the servo amplifiers, DC motor units, and a reduction gear tacho unit.

\subsubsection{Tacho generator}

Tacho generator is an electronic device that converts mechanical energy to electrical energy. The measurement results are showing revolutions per min on an analog scale meter. Sensor faults tacho generators occurs at mechanical variables, such as bearings or bearings, brush wear, shaft, extrusion and rotor misalignment. Faults that occur in mechanical variables can cause minor faults on the system in the form of bias errors and sensitivity [10].

\subsubsection{Servo amplifier}

The servo amplifier is an amplifier that can signal an error processing to correct for the difference between the input signal (reference) with the feedback signal (feedback) so that it can produce the desired output. Fault on the actuator occurs because of the supply voltage, the decrease in the performance of the amplifier and blockages. The fault relates to errors loss of effectiveness (partial loss of hydraulic or pneumatic pressure) and refractive fault in the actuators [11].

\subsection{Passive fault-tolerant system}

An improved performance and security required in system control. The feedback control system produces a less stable performance when used on complex systems if damage occurs on actuators, sensors, and other components. The solution to fix the problem a control design that can tolerate faults, so that system stability is maintained. Fault-Tolerant Control System (FTCS) is a control system that can tolerate errors in a system so that the system can achieve the desired performance [12]. FTCS can be divided into two types, namely Passive Fault Tolerant Control System (PFTCS) and Active Fault Tolerant Control System (AFTCS). AFTCS is a control system that reacts to component failure with reconfiguring control so that the system stability and performance can be maintained [13]. Meanwhile, PFTCS is a control system that is designed to maintain the system from a failure that occurred. The difference between the two types of FTCS is in the design of Reconfigurable Controller (RC) and Fault Detection and Isolation (FDI), which are only used on AFTCS.

The advantages of the PFTCS system are predefined faults so that the system can be designed to be a robust controller of faults, and there is no delay due to RC and FDI designs that usually happen on AFTCS. However, if a fault occurs outside the specified value, the 
system performance will deteriorate. Therefore, the PFTC design requires observers to be able to estimate errors so that system performance can be maintained [1].

\section{Research methods}

\subsection{Modular DC motor servo system}

The selection of the model performed with the parametric method based on input data and output data from the plant have been obtained. The modular servo system of DC motor is designed as shown in the image for input and output.

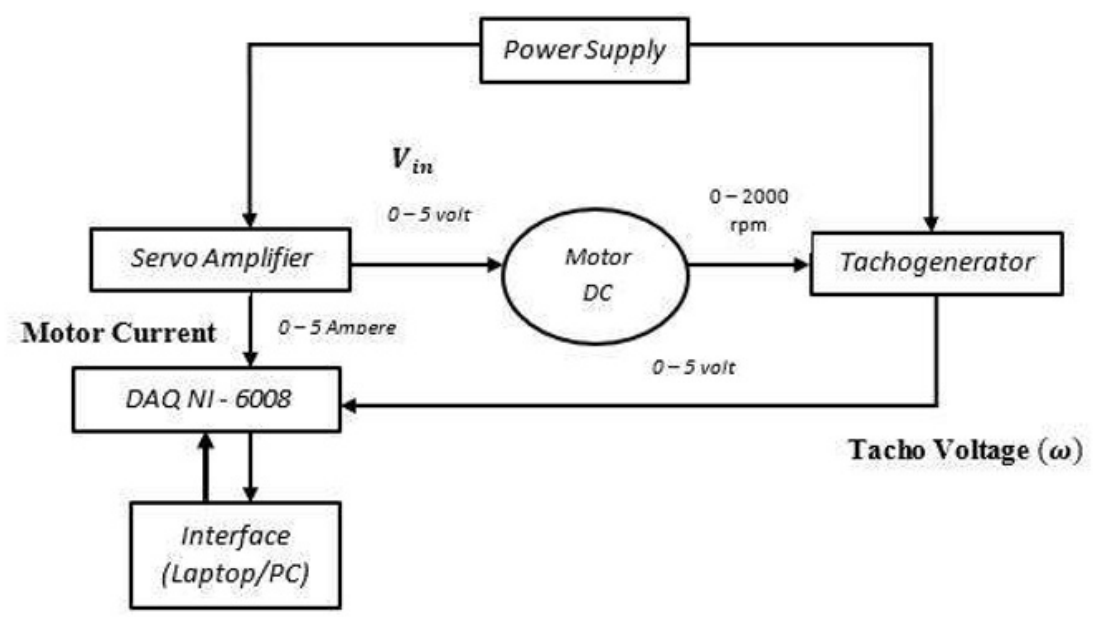

Fig 2. Block diagram of servo modular system set up

Based on Figure 2, a system of control is located on the computer/laptop or user interface. The control system to set the input signal on motor DC motor, because speed control of the DC motor is influenced by the magnitude of the voltage input is given. NIDAQ 6008 is used for communication between computers and plant. Besides, NI-DAQ 6008 is needed as a media to convert digital to analog signals. The data used in this research is input data voltage and output data in the form of the value of the speed and the current. Based on the modeling that was done then obtained state-space system as follows in Equation (1) and Equation (2),

$$
\begin{gathered}
{\left[\begin{array}{c}
\dot{\omega} \\
i
\end{array}\right]=\left[\begin{array}{cc}
-\frac{1}{\tau_{i}} & 0 \\
0 & -\frac{1}{\tau_{i}}
\end{array}\right]\left[\begin{array}{c}
\omega \\
i
\end{array}\right]+\left[\begin{array}{c}
\frac{K_{i}}{\tau_{i}} \\
\frac{K_{i}}{\tau_{i}}
\end{array}\right]} \\
{\left[\begin{array}{c}
\dot{\omega} \\
i
\end{array}\right]=\left[\begin{array}{cc}
-\frac{1}{5} & 0 \\
0 & -\frac{1}{0.01}
\end{array}\right]\left[\begin{array}{c}
\omega \\
i
\end{array}\right]+\left[\begin{array}{c}
\frac{2.8}{5} \\
\frac{0.99}{0.01}
\end{array}\right] V}
\end{gathered}
$$




\subsection{The Design of Feedback Control Systems}

$$
\begin{aligned}
& \dot{x}=A x+B u \\
& y=C x+D u
\end{aligned}
$$

Which the amount of error,

$$
\begin{gathered}
\dot{e_{c}}=\omega_{\text {ref }}-\omega \\
\dot{e_{c}}=\omega_{\text {ref }}-C_{r} \cdot x
\end{gathered}
$$

Equation (4) and Equation (6) are changed in the form of a matrix as Equation (7), follows:

$$
\left[\begin{array}{c}
\dot{x} \\
\dot{e}_{c}
\end{array}\right]=\left[\begin{array}{cc}
A & 0 \\
C_{r} & 0
\end{array}\right]\left[\begin{array}{c}
x \\
e_{c}
\end{array}\right]+\left[\begin{array}{c}
B \\
0
\end{array}\right] u+\left[\begin{array}{l}
1 \\
0
\end{array}\right] \omega_{r e f}
$$

Selected the desired pole as follows,

$$
\begin{aligned}
& p 1=-15+5 i \\
& p 2=-15-5 i \\
& p 3=20
\end{aligned}
$$

So, it is with the method of pole placement controller gain value is obtained in Equation

(8), and Equation (9):

$$
\begin{gathered}
K_{c}=\left[\begin{array}{ll}
K_{p} & K_{i}
\end{array}\right] \\
K_{c}=\left[\begin{array}{lll}
224.99 & 19 & 2.5
\end{array}\right]
\end{gathered}
$$

\subsection{The Design of Observers}

The observer design is used to estimate existing faults, by developing system state-space equations. State errors of sensors and actuators are each symbolized by fa and fs. The statespace containing an actuator error can be written in Equation (10) and Equation (11),

$$
\begin{gathered}
\dot{x}=A x+B u+F_{a} f_{a} \\
y=C x+F_{s} f_{s}
\end{gathered}
$$

The addition of the state needed to move the functions of the Fs to the state space,

$$
\dot{z}=A_{z}(y-z)+z
$$

Equation (11) is substituted with Equation (12) so that it is obtained,

$$
\dot{z}=A_{z}\left(C x+F_{s} f_{s}\right)+z
$$

Equation (11) and Equation (13) can be changed into a state-space such as Equation (14) and Equation (15),

$$
\left[\begin{array}{c}
\dot{x} \\
\dot{z}
\end{array}\right]=\left[\begin{array}{cc}
A & 0 \\
A_{z} C & A_{z}
\end{array}\right]\left[\begin{array}{l}
x \\
z
\end{array}\right]+\left[\begin{array}{c}
B \\
0
\end{array}\right] u+\left[\begin{array}{cc}
F_{a} & 0 \\
0 & A_{z} F_{s}
\end{array}\right]\left[\begin{array}{l}
f_{a} \\
f_{s}
\end{array}\right]
$$




$$
y=\left[\begin{array}{ll}
0 & 1
\end{array}\right]\left[\begin{array}{l}
x \\
z
\end{array}\right]
$$

To obtain the structure matrix expansion required the observer through new state-space Equation (16) and Equation (17).

$$
\bar{x}=\bar{A} \cdot \bar{x}+\bar{B} \cdot u+\bar{F} \bar{f}
$$

$$
\bar{y}=C \bar{x}
$$

The equation of state and output equations of containing error actuators and sensors used to design observer Equation (18) and Equation (19),

$$
\begin{gathered}
\hat{\bar{x}}=\bar{A} \cdot \hat{\bar{x}}+\bar{B} \cdot u+\bar{F} \hat{\bar{f}}+K_{e}(\bar{y}-\hat{\bar{y}}) \\
\hat{\bar{y}}=C \hat{\bar{x}}
\end{gathered}
$$

State containing the gain added to estimate the actuator and sensor faults (L),

$$
\hat{f}=T_{s} L_{e}+\hat{f}
$$

So, from Equation (16) and Equation (17) can be converted into Equation (21),

$$
\begin{gathered}
{\left[\begin{array}{l}
\hat{\bar{x}} \\
\hat{f}
\end{array}\right]=\left[\begin{array}{cc}
\bar{A} & \bar{F} \\
0 & I
\end{array}\right]\left[\begin{array}{l}
\hat{\bar{x}} \\
\hat{\hat{f}}
\end{array}\right]+\left[\begin{array}{l}
\bar{B} \\
0
\end{array}\right] u+\left[\begin{array}{c}
K \\
L T_{s}
\end{array}\right] e} \\
\hat{\bar{y}}=\left[\begin{array}{ll}
\bar{C} & 0
\end{array}\right]\left[\begin{array}{l}
\hat{\bar{x}} \\
\hat{f}
\end{array}\right]
\end{gathered}
$$

With $\hat{\bar{x}} \hat{f} \hat{\bar{y}}$, are the results of the estimation of augmented state errors and output. $K$ is the proportional gain of the observer and $L T_{S}$ is the integral gain from the observer. The gain observer is used to guarantee the stability of the dynamical error estimation.

The next step that must be done after determining the design of a robust observer is to determine the generalized plant. A generalized plant is formed from the knowledge of the given plant and the weighting transfer function that represent design objectives. The $H_{\infty}$ design procedure is then applied to the generalized plant to design a robust controller.

The generalized plant state-space equation is obtained by six states, namely $\widehat{x_{z}}, \widehat{f}, \widehat{x}, x, e_{C}, z$ so that the equation can be written as follows, in Equation (22).

$$
\left[\begin{array}{c}
\dot{\hat{x}} \\
\dot{\hat{x}} \\
\dot{\hat{f}} \\
\dot{x} \\
\dot{e_{C}} \\
\dot{z}
\end{array}\right]=\left[\begin{array}{cccccc}
0 & 0 & -B K_{p}+A_{a} & 0 & -B . K_{i} & 0 \\
-I & 0 & 0 & A_{z} C & 0 & 0 \\
0 & 0 & E_{a} & 0 & 0 & 0 \\
0 & 0 & -B . K_{p} & A & -B . K_{i} & 0 \\
-C_{r} & 0 & 0 & 0 & 0 & 0 \\
A_{1} C_{r} B K_{p} & 0 & 0 & -A_{1} C_{r} A-A_{2} C_{r} & A_{1} C_{r} B K_{i} & -A_{3}
\end{array}\right]\left[\begin{array}{c}
\hat{x} \\
\widehat{x_{z}} \\
\hat{f} \\
x \\
e_{C} \\
Z
\end{array}\right]+\left[\begin{array}{cc}
0 & 0 \\
0 & L_{x x} \\
0 & L_{x x z} \\
0 & 0 \\
I & 0 \\
A_{2} & 0
\end{array}\right]\left[\begin{array}{c}
y_{r} \\
e_{L}
\end{array}\right]
$$




\section{Results and discussions}

\subsection{Test tracking setpoint realtime}

PFTC algorithm is applied in realtime to the MS150 DC servo. Based on the LQR and robust values that have been obtained it will be used for the setpoint tracking test using Labview 2013 software that has been connected with the MS150 DC with the help of the NI-DAQ 8000 interface. Changes in the setpoint value are made from the speed of $735 \mathrm{rpm}$ to $1095 \mathrm{rpm}$ or from a voltage of $2 \mathrm{~V}$ to $3 \mathrm{~V}$ at $8396 \mathrm{~ms}$. So from the two graphs, it can be seen that systems with robust control signals have more accurate tracking responses compared to the results of the LQR control system response with a criterion of $2 \%$, systems with robust control produce settling time of $2244 \mathrm{~ms}$.

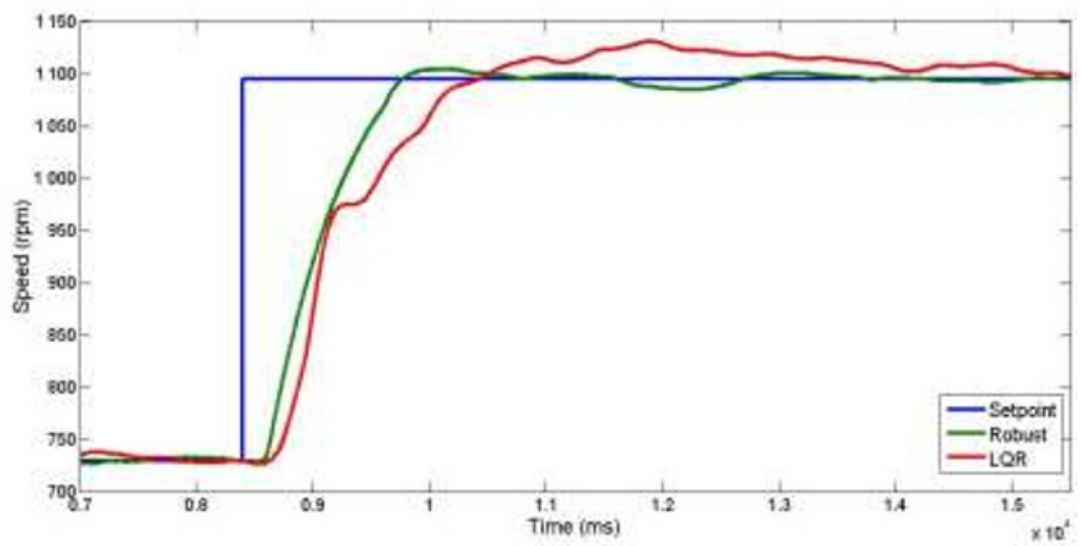

Fig 3. Test the Realtime Set Point Tracking with Labview software

\subsection{Observer estimation test results in realtime}

The estimation stage test results are carried out with the help of Labview 2013 software to determine the observers performance. Estimation test is done by comparing the response of the system when given a fault with the addition of observer and without the observer.

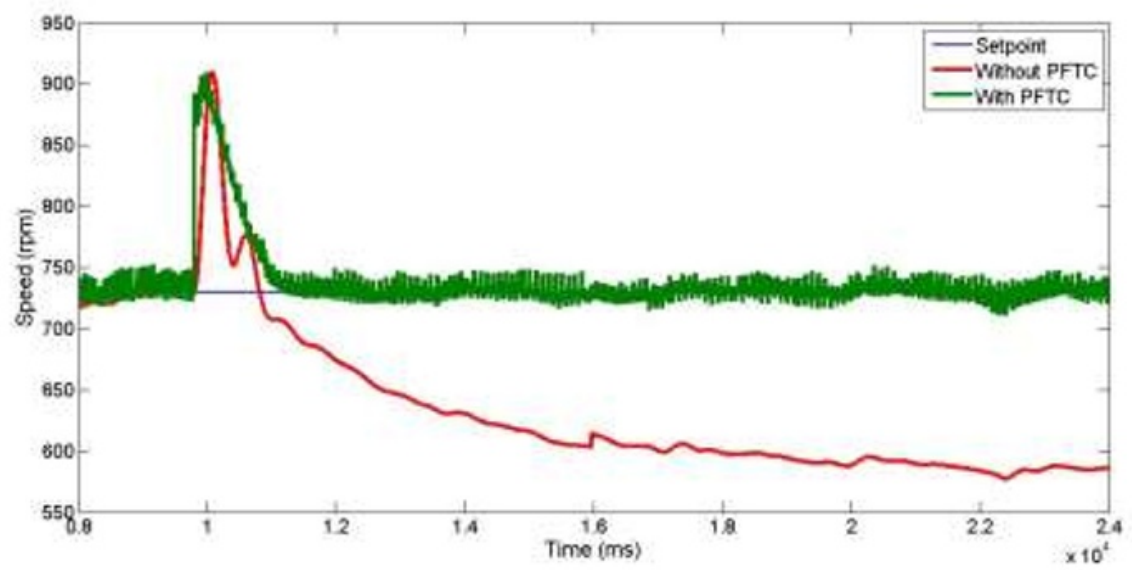

Fig 4. Observer Estimation Results 
In Figure 4, it can be seen that the red graph shows the system's response when given an error without an observer. On systems without FTC, it can be seen that after experiencing an error the system response cannot return to the setpoint value and results in a steady-state error of $19.15 \%$. Whereas in an FTC system, the estimated error results can return to the setpoint after being given an error. So, it can be concluded that the FTC has been able to work properly to estimate the magnitude of errors that occur.

\subsection{Fault sensor and actuator test in realtime}

There are several variations of error values from actuators and sensors that will be applied to the MS150DC motor system. The real plant error test is carried out by giving errors to the actuator components and system sensors with the help of Labview 2013 software. Actuator fault is given on the control signal. Sensor fault is given on the measurement signal.

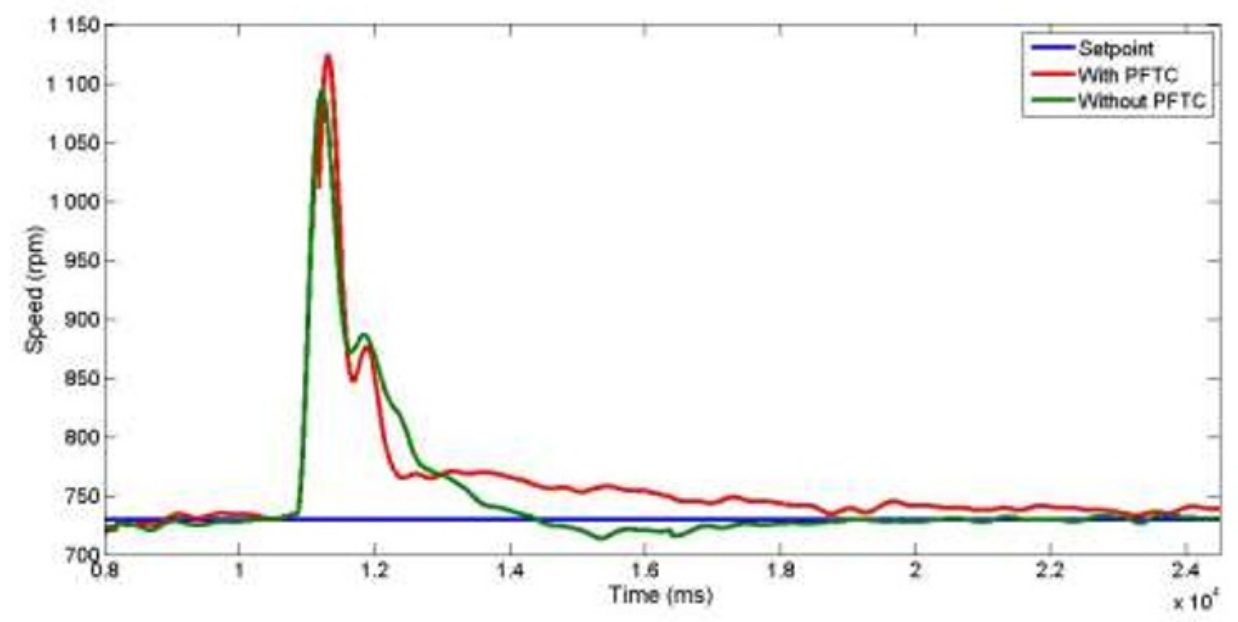

Fig 5. Actuator fault response system with LQR gain.

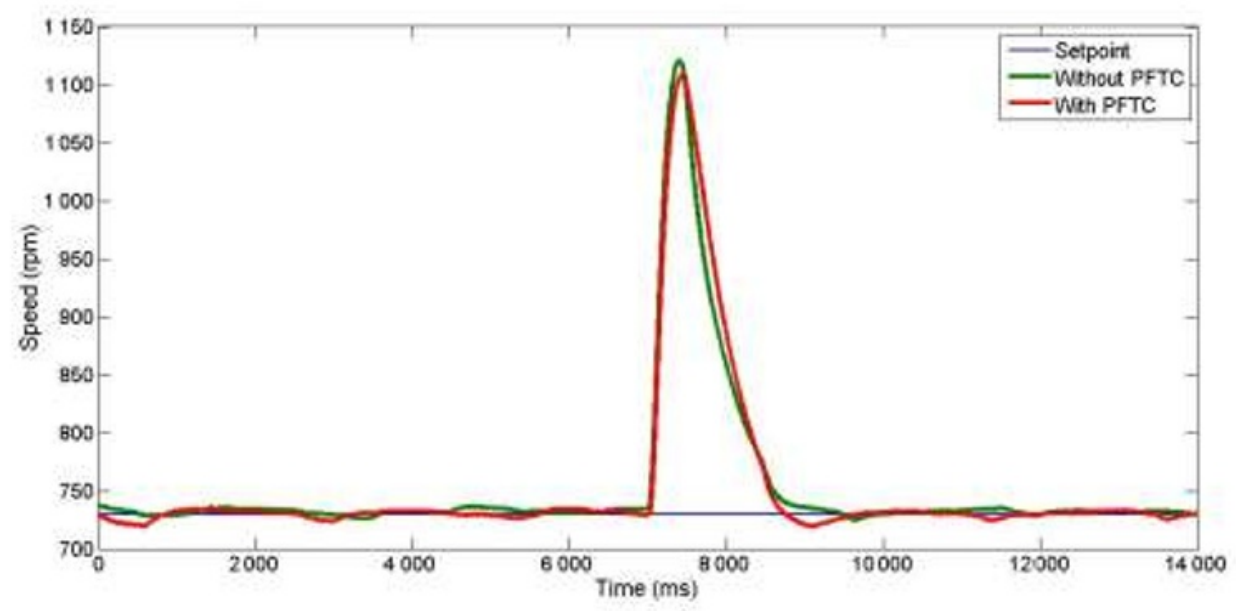

Fig 6. Actuator fault response system with a robust gain. 
The state feedback control system without FTC can maintain performance without adding FTC. This can be seen in Figures 6 and Figure 7 without FTC graphs produce a faster response than when the system was added FTC. The use of robust methods is better when estimating errors that occur than the LQR method. Systems without FTC produce higher overshoot compared to systems with FTC but systems without FTC can return responses to steady-state after being subjected to an error.

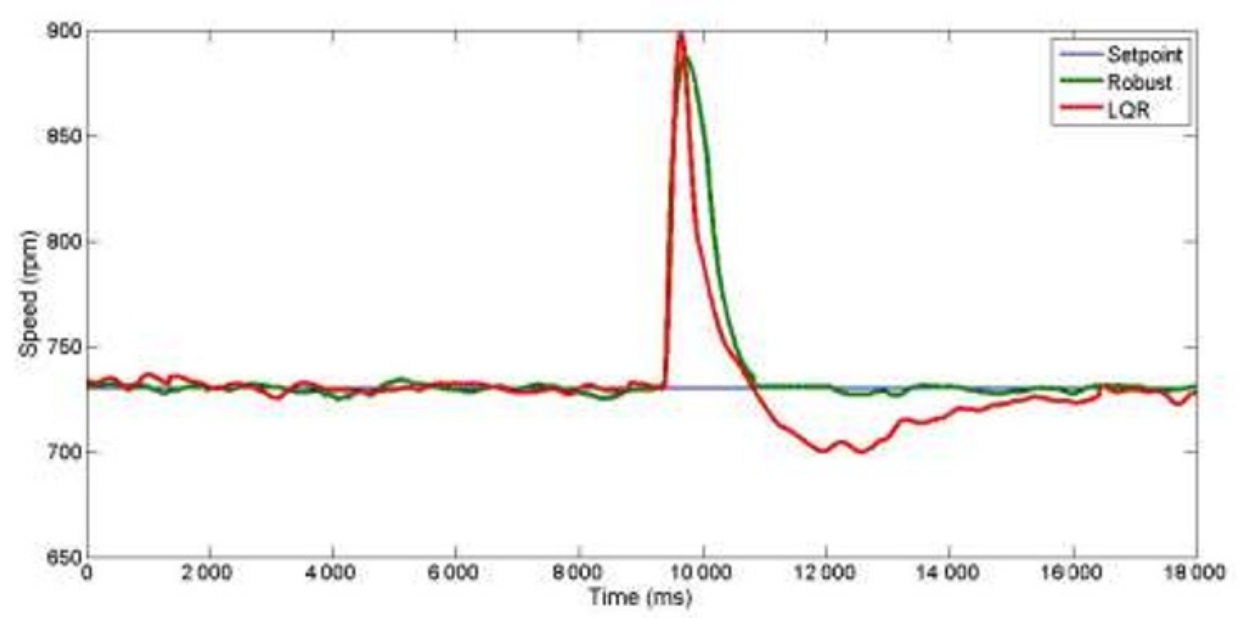

Fig 7. Sensor fault response.

\section{Conclusion}

The response obtained when the FTC algorithm is implemented shows that the system designed can work because it can estimate the bias error in the actuator and sensor components so that the system response can return to a steady-state value. The response generated in real-time is not as good as the response of simulation results because when the FTC algorithm is implemented directly (real-time) there is a direct measurement factor and the appearance of disturbance on the measurement so that it can affect the estimated output of the observer. In the real plant test, there is a difference between the model used by the observer and the real system. However, in the simulation, the equation between the model and the real system is the same so that it produces better response results than the real plant response results.

Based on the design and implementation that has been done, the maximum error that can be overcome for the actuator error is $100 \%$ or when the DC motor servo system MS15DC has been unable to rotate or in other words, the system has a major error, the FTC algorithm cannot correct. Actuator fault in real-time performance tests can be estimated using a state feedback control system without FTC. Real-time PFTC testing can estimate actuator and sensor errors. The magnitude of the error of the actuator and sensor bias is around $0.2 \mathrm{~V}, 0.4 \mathrm{~V}$, and $0.6 \mathrm{~V}$. Systems without PFTC have a settling time of $7.030 \mathrm{~ms}$ for LQR control system responses and $2502 \mathrm{~ms}$ for robust control system responses. 


\section{References}

1. M. Dybkowski, K. Klimkowski, Autom. - J. Control. Meas. Electron. Comput. Commun., 57,3:638-647(2017).

https://www.semanticscholar.org/paper/Fault-Tolerant-Control-Structure-forInduction-Dybkowski-Klimkowski/4c655a796b38501e691d32686d934bbd4caa9c5d

2. G.G. Rigatos, Electr. Power Syst. Res., 79,11:1579-1592(2009). https://www.sciencedirect.com/science/article/abs/pii/S0378779609001473

3. H. Tohidi, K. Erenturk, S. Shoja-Majidabad, Control Eng. Appl. Informatics, 19,1:4958(2017).

http://ceai.srait.ro/index.php?journal=ceai\&page=article\&op=viewFile\&path[]=3395 $\underline{\text { \&path }[]=1428}$

4. S. Fan, J. Zou, Sensor Fault detection and fault tolerant control of induction motor drivers for electric vehicles, Proc. IEEE 7th Power Electron. Motion Control, (Harbin, China, 2012), pp. 1306-1309.

https://ieeexplore.ieee.org/document/6259046

5. H. Noura, D. Sauter, F. Hamelin, D. Theilliol, IEEE Control Syst. Mag., 20,1:3349(2000).

https://ieeexplore.ieee.org/document/823226

6. P. Dobra, G. Lazea, D. Moga, M. Tru, Acta Electrotehnica 43,1:43-48(2002).

https://ie.utcluj.ro/files/acta/2002/Number\%201/Paper06_Dobra.pdf

7. D.U. Campos-Delgado, S. Martínez-Martínez, K. Zhou, IEEE/ASME Trans. Mechatronics, 10,4:419-427(2005).

https://ieeexplore.ieee.org/document/1512165

8. H. Jerrar, A. El Marjani, E.M. Boudi, H. Kim, P. Lorenz, J.P. Meyer, International Review on Modelling and Simulations 8,4:410-417(2015).

https://www.praiseworthyprize.org/jsm/index.php?journal=iremos\&page=article\&op= view\&path[]=17662

9. A. Hughes, B. Drury, Electric Motors and Drives. Oxford: Newnes (2019). https://www.elsevier.com/books/electric-motors-and-drives/hughes/978-0-08-1026151

10. D.U. Campos-Delgado, D.R. Espinoza-Trejo, E. Palacios, IET Electric Power Applications 2,2:121-134(2008).

https://ieeexplore.ieee.org/document/4480891

11. K. Ogata, Modern Control Engineering, USA: Prentice Hall (2010). http://sharif.edu/ salarieh/Downloads/Modern\%20Control\%20Engineering\%205th\%2 0Edition.pdf

12. Y. Zhang, J. Jiang, Ann. Rev. Control. 32,2:229-252(2008). https://www.sciencedirect.com/science/article/pii/S1367578808000345

13. Q. Zhao, Fault tolerant control systems design, [Thesis], The University of Western Ontario, Canada, (1999).

http://citeseerx.ist.psu.edu/viewdoc/download?doi=10.1.1.428.201\&rep=rep1\&type=p $\underline{\mathrm{df}}$ 